\title{
ALGUNOS ASPECTOS DEL PROCESO DE CONSTRUCCIÓN DEL MUSEO GUGGENHEIM BILBAO. BILBAO/ESPAÑA
}

\author{
(SOME ASPECTS OF THE CONSTRUCTION PROCESS OF THE GUGGENHEIM BILBAO \\ MUSEUM. BILBAO/SPAIN)
}

César Caicoya Gómez-Morán, Arquitecto

ESPAÑA

Fecha de recepción: 7-X-97

\section{RESUMEN}

Se describen - de manera breve-algunos aspectos del proyecto y construcción del Museo Guggenheim Bilbao, planteando y resolviendo los distintos problemas que se presentaron durante el proceso constructivo (urbanisticos, funcionales, arquitectónicos, etc.).

Se hace hincapié, asimismo, en la complejidad geométrica del proyecto y de su solución final.

\section{SUMMARY}

This paper contains a brief description of some aspects of the project and the construction of the Guggenheim Bilbao Museum. Arrangements and solutions for the different problems (urbanistic, fonctional, architectural, etc.) arised along with the building process are described.

The geometrical complexity of the project and its final solution are emphasized.
El proyecto del Museo Guggenheim Bilbao surge de una conjunción de intereses. Por un lado las instituciones del País Vasco, tras un rápido proceso de desindustrialización del País, programan, en los últimos años de la década de los 80 , un cambio de actividad dirigido a impulsar el sector servicios apoyándose en transformaciones del tipo comunicaciones (Aeropuerto, de S. Calatrava, Metro, de N. Foster, Estación Intermodal, de J. Stirling, Superpuerto, puentes sobre la ría de Bilbao, etc.), en las nuevas tecnologías (Parques Tecnológicos, de Zamudio, Miramón y Miñano)y, por último, en la cultura(Museo Guggenheim, de F. Gehry, Palacio de la Música y Congresos de Bilbao, de F. Soriano, Palacio de Congresos de San Sebastián, de R. Moneo, etc.). Una de las locomotoras catalizadoras de este cambio se pensó que fuera el Museo Guggenheim Bilbao.

Por otra parte, la Solomon R. Guggenheim Foundation, con su recién acabada ampliación de la $5^{\mathrm{a}}$ Avenida $(\mathrm{Ch}$. Gwathmey) y la reforma de la sede del Soho (A. Isozaki), no tenía capacidad expositiva suficiente para exponer su magnífica colección de arte moderno y contemporáneo (un $94 \%$ de sus fondos estaban almacenados), por lo que llevaba unos años gestionando una alianza en Europa (Salzburgo, Venecia, Berlín).
Así, en 1991 se llega a un acuerdo por el que, en síntesis, las instituciones del País Vasco aportan el inmueble y una colección propia y la S. R. Guggenheim Foundation, aporta su conocimiento museográfico y su colección.

El interés de las dos partes no es sólo el tener un Museo, sino activar todo un proyecto cultural que regenere el tejido urbano de Bilbao y, por otra parte, sirva de emblema internacional del nuevo rumbo que toma el País Vasco.

Tras un rápido concurso entre tres arquitectos (Arata Isozaki, Coop. Himmenblau y F. Ghery) seleccionados como representantes de tres continentes, el jurado, reunido en Frankfurt y presidido por Heinrich Klotzle, otorga el $1^{\text {er }}$ premio al proyecto de Frank O. Gehry.

El proyecto ganador responde perfectamente a los requerimientos, a saber:

- Urbanísticamente da respuesta al complejo terreno, de frontera entre el ensanche del siglo XIX y XX y la ría postindustrial. Por una parte, se genera una gran plaza peatonal que enlaza con la ciudad construida, sobre la que se elevan unos clásicos edificios de piedra y raseo de cemento. Por otra parte, se proyecta un paseo de ribera colindante con la 
ría, rodeado de una lámina de agua sobre la que se elevan unos curvos edificios de titanio. La plaza y el paseo públicos, se enlazan por medio de una rampa. Además, se proyecta una gran torre de piedra de geometría compleja, resuelta con piedras talladas con formas curvas, al otro lado del enorme tablero del puente de La Salve, de tal modo que se absorbe y resuelve el impacto estético y urbanístico de este puente.

- A pesar del impacto volumétrico del tamaño del edificio $\left(24.000 \mathrm{~m}^{2}\right.$ ) y con espacios de gran altura (hasta $54 \mathrm{~m}$ ), al resolver el programa por medio de la agregación de volúmenes independientes, se conserva la escala urbana y humana.

- Funcionalmente resuelve el programa museográfico con brillantez, al generar un microcosmos compuesto por los diversos cuerpos edificatorios definidos por el cliente (diversos tipos de galerías de exposiciones, auditorio, restaurante, tienda, administración, conservación, etc.), enlazados entre sí mediante rótulas de cristal (complejos muros cortina) que permiten percibir el ambiente

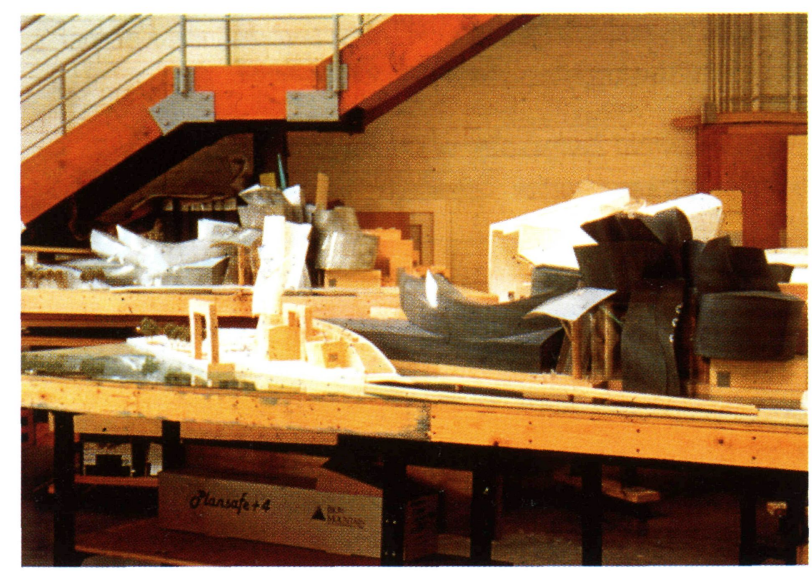

Trabajo en maquetas en el estudio de F.O. Gehry.

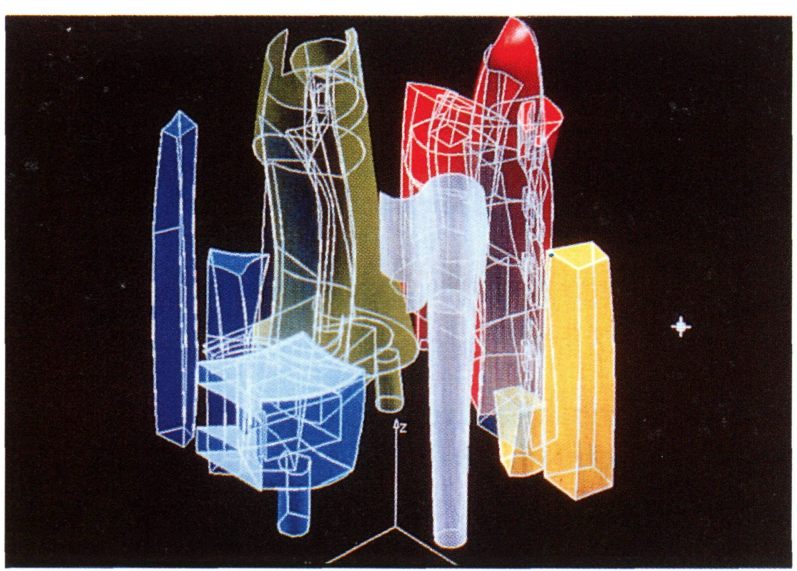

Trabajo en Catia: modelando volúmenes.

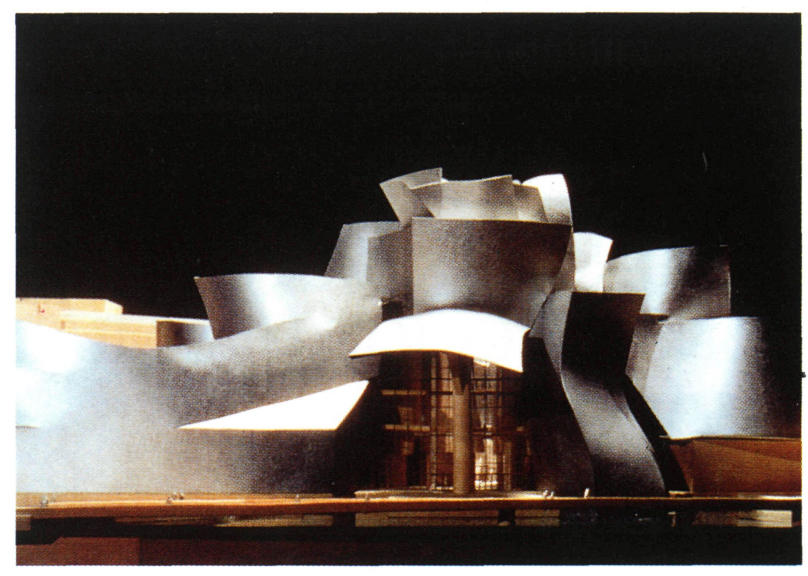

Maqueta final: obtenida por CAD-CAM.

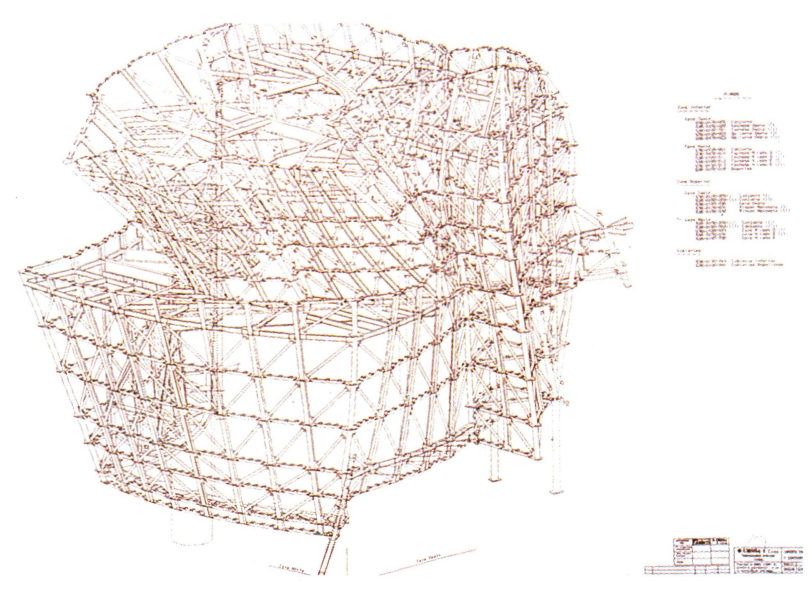

Diseño y cálculo de estructura: programa BOCAD

exterior (ciudad, ría, clima, etc.), todo ello compuesto alrededor del gran atrio central que vertebra y ordena espacialmente este microcosmos.

- El lenguaje arquitectónico utilizado dista tanto de los epígonos de la modernidad como del melancólico recurso a la memoria histórica. Así, se asume una arquitectura no dogmática sino contingente, en la que se acepta el natural pluralismo humano. A base de un constante "probar" por el procedimiento de iteraciones sucesivas con maquetas y en ordenador (Catia), se consigue la frescura, lo natural y espontáneo de lo aparentemente improvisado transmitiendo una imagen de caos, optimismo y belleza que ha calado muy hondo, tanto en el ciudadano de a pie (popular) como en el más exigente crítico de arte y arquitectura (culto), aglutinando, de este modo, una nueva imagen iconográfica local e internacional pretendida en el proyecto cultural.

El proyecto final tiene una gran complejidad geométrica (superficies no regladas, tanto interior como exteriormente); constructiva (grandes partes del proyecto se han construido mediante CAD-CAM, como, por ejemplo, la estructura metálica principal, las estructuras secundarias 
y terciarias o la piedra curva) y tecnológica (sin el apoyo de programas de gran capacidad matemática y gran velocidad o sin la utilización de máquinas de control numérico para distintas fases de la obra, no hubiera sido posible la construcción de este edificio, en el plazo y costo prefijados).

Para resolver estas complejidades descritas sumariamente (además de la de gestión del proyecto y de las obras) participaron diversos consultores externos, con la firma de arquitectura, consultoría e ingeniería IDOM, quien se responsabilizó, junto con la ayuda de los diversos contratistas y en una muy estrecha relación con el arquitecto de diseño, de hacer realidad lo que al inicio no parecía más que un sueño. Para ello, IDOM organizó diversos equipos de Arquitectura con una coordinación general (César Caicoya), de Ingeniería (J.M. Asumendi) y de Gestión (Luis Rodríguez), formando un equipo de personas que mantuvo un tamaño medio de 45 técnicos durante 5 años, llegando en los momentos punta de trabajo a 150 profesionales, que asumieron el reto con entusiasmo y dedicación, encontrando soluciones a problemas nuevos; investigando nuevas técnicas de construcción y ensayando nuevos materiales; no conformándose con soluciones meramente aceptables, sino anhelando la máxima calidad posible, tanto en diseño (del orden de 16.000 faxes intercambiados con la oficina de Frank O. Gehry), como en la construcción (con parte de equipo de IDOM fijo a pie de obra), para obtener el Museo que todos deseábamos.

El prestar una total y obsesiva atención, se ha demostrado como esencial para encontrar buenas soluciones, al, por tantos anunciado, como un proyectobellísimoen maquetas, pero inconstruible. Asimismo, la transmisión de esta dedicación a todos los contratistas y subcontratistas ha sido

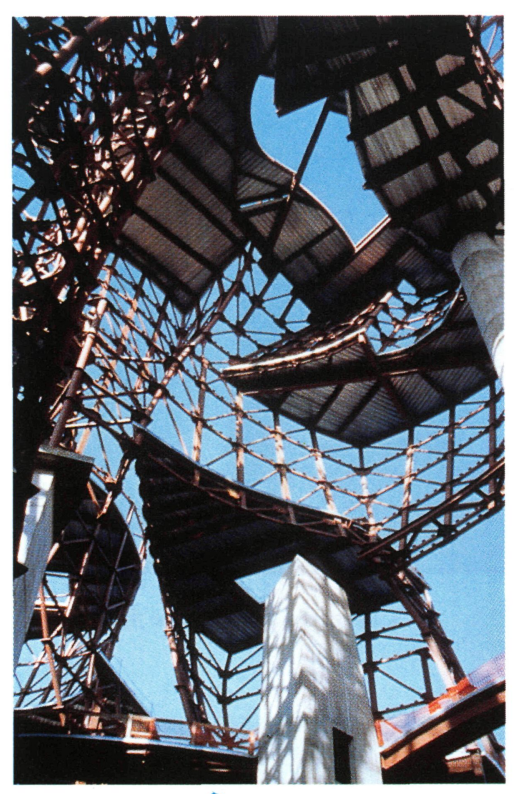

Montaje de la estructura metálica.

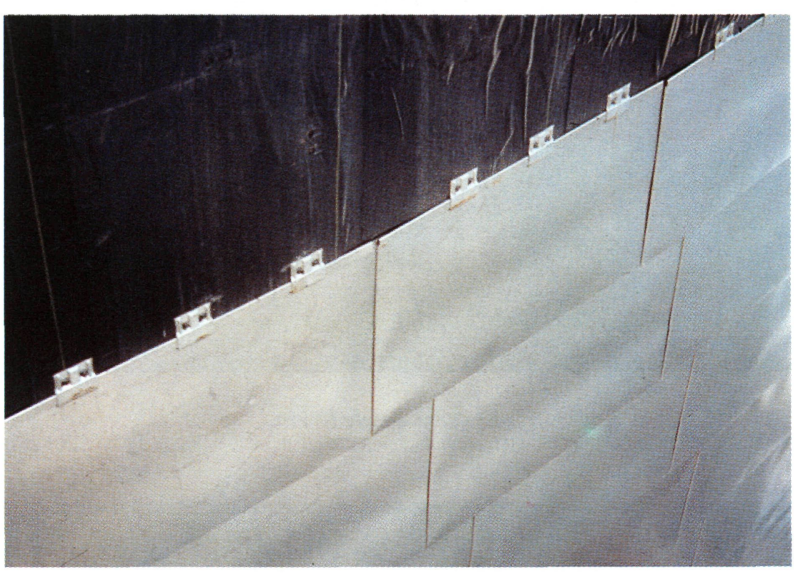

Montaje del titanio en fachadas.

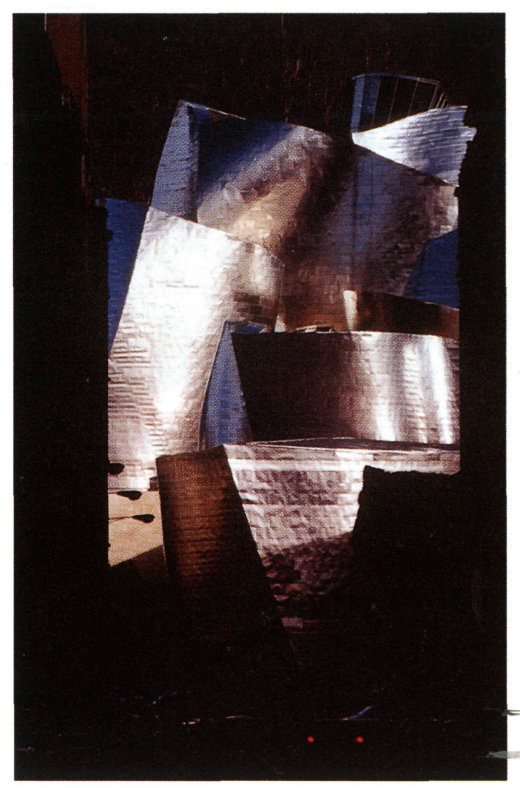

I'ista del Museo desde la ciudad. 


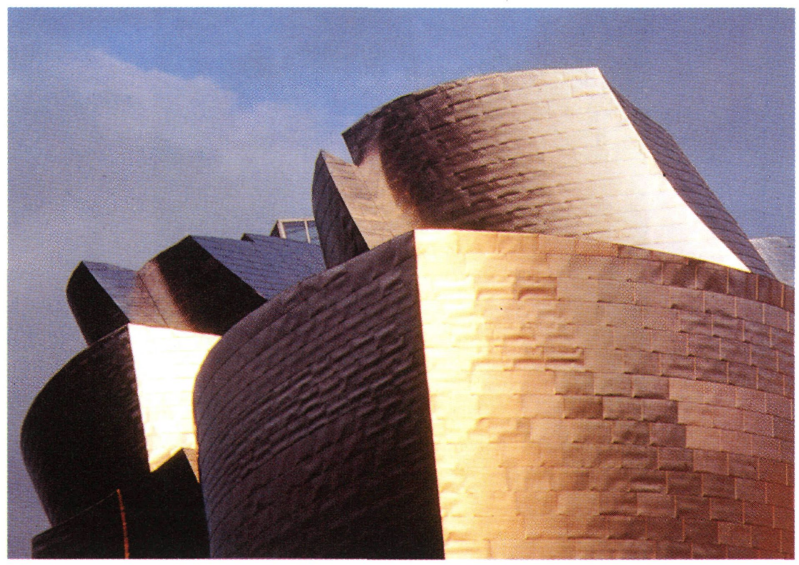

Cuerpos de titanio: la flor.

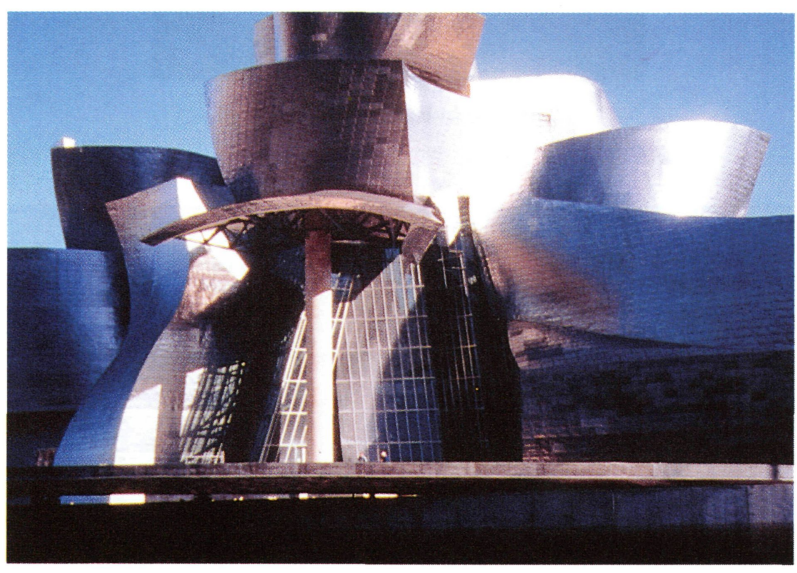

El atrio desde la ría.

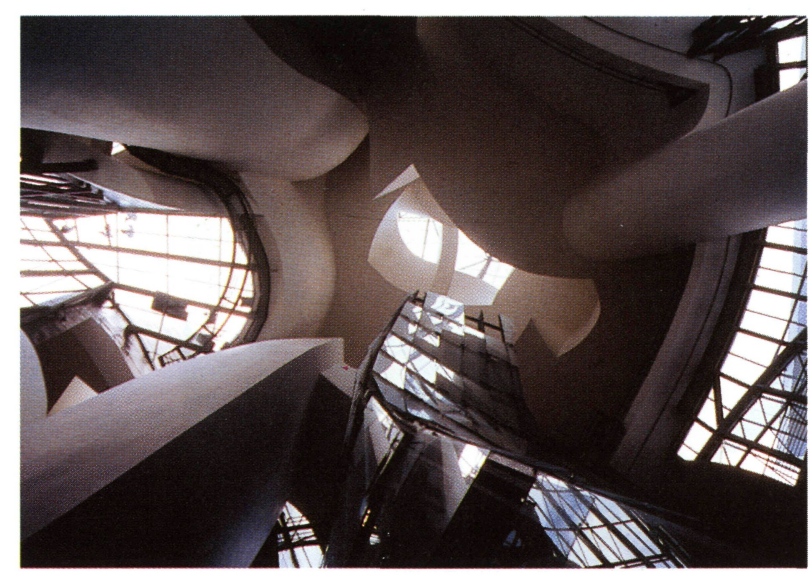

Vista interior del atrio.

fundamental, pues el hallazgo de nuevas soluciones a nuevos problemas se debe, en buena parte, a su gran involucración en el proyecto. Por otra parte, aesta constante tensión ha contribuido no sólo el reto de construir un sueño nunca antes ejecutado, sino las muchas restricciones que han existido, entre las que se pueden contar como muy importantes el plazo (inaugurar en 1997) y el costo (10.000

(c) Consejo Superior de Investigaciones Científicas Licencia Creative Commons 3.0 España (by-nc)

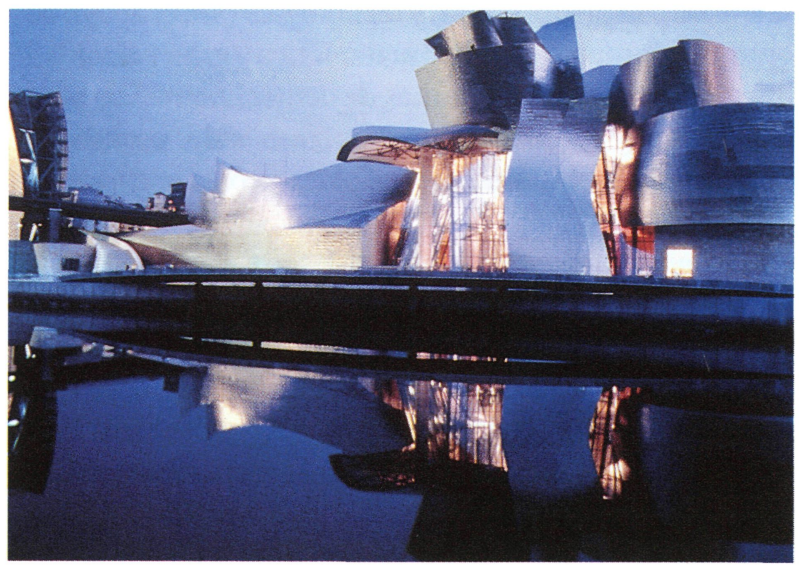

Vista del Museo desde la ría.

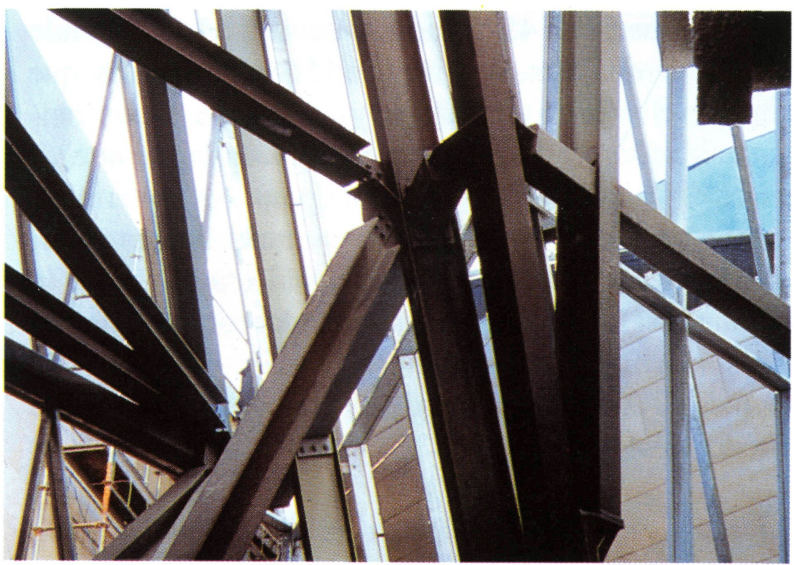

Un muro cortina: rótula de cristal.

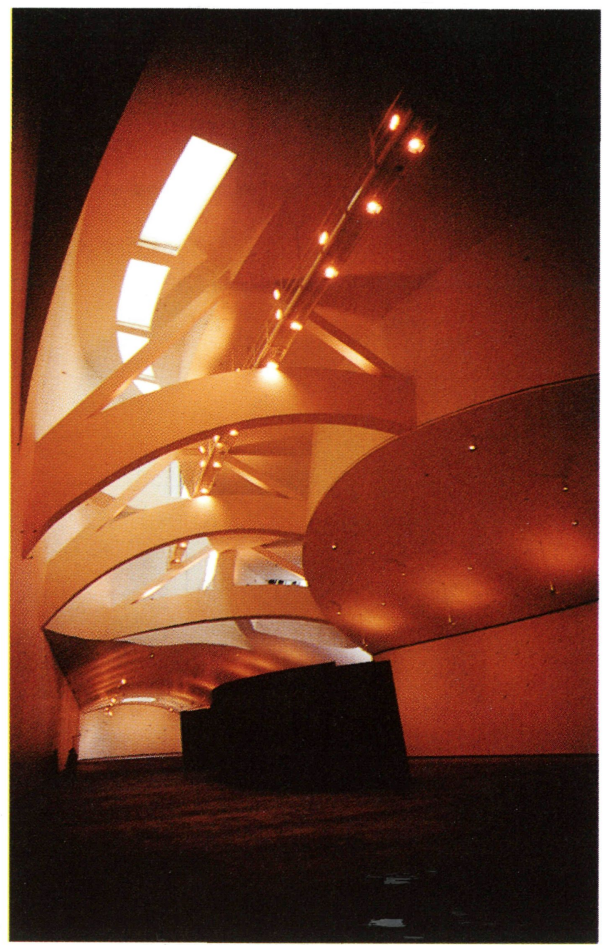

Vista de la galería de exposiciones temporales. 
millones de pesetas para el edificio). Todos ellos muy limitados, escasos y perfectamente definidos y exigidos por el cliente desde el inicio, de tal modo que ha conseguido liderar este proyecto obteniendo lo mejor de cada una de las

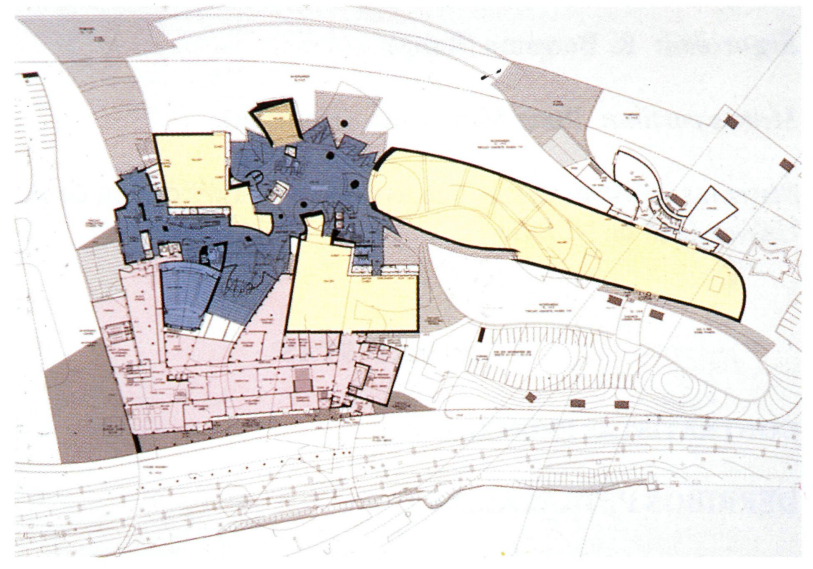

Planta baja, en amarillo: galerías de exposición.

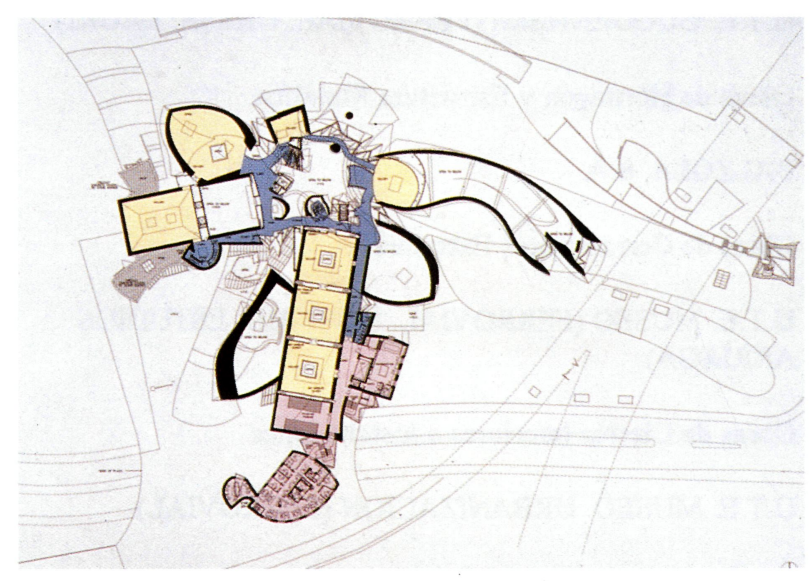

Planta segunda, en rosa: servicios internos. partes involucradas en él, sin permitir, en ningún momento, a lo largo de los cinco años que ha durado el proyecto y la construcción, que nadie rebajara la tensión en cuanto a la calidad, el plazo y el costo prefijados.

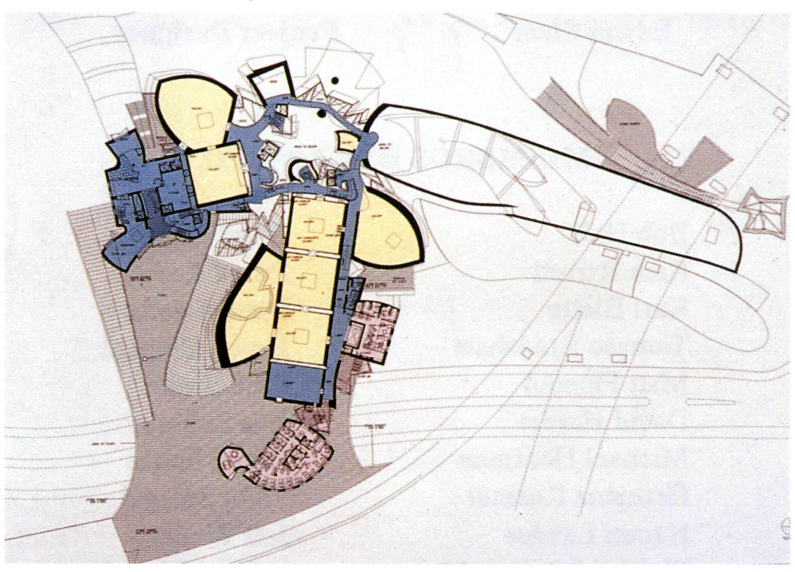

Planta primera, en azul: locales y espacios de comunicación.

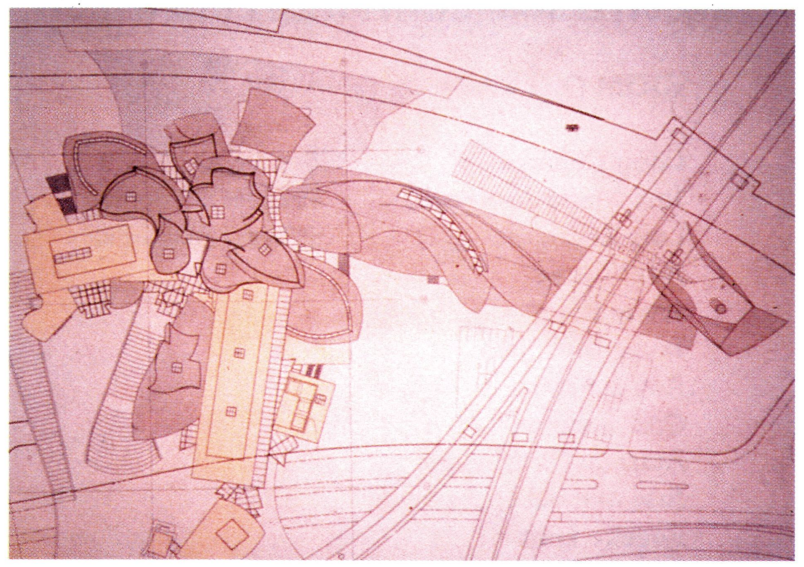

Planta cubiertas.

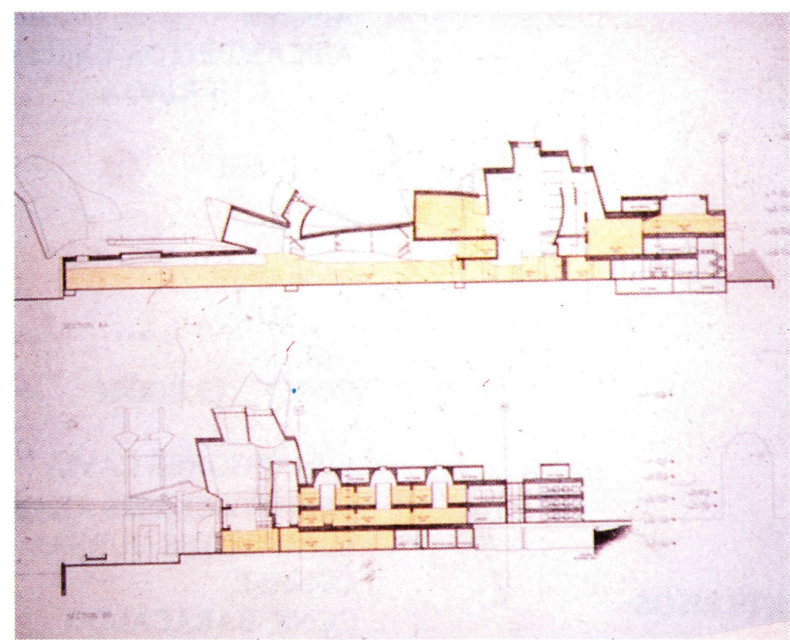

Secciones: 


\section{EQUIPO DE PROYECTO}

\author{
Frank O. Gehry \\ Randy Jefferson \\ Vano Haritunians \\ Douglas Hanson \\ Edwin Chan
}

\author{
Design Principal \\ Project Principal \\ Project Manager \\ Project Architect \\ Project Designer
}

\section{MIEMBROS DEL EQUIPO}

Bob Hale

Rich Barrett

Karl Blette

Tomaso Bradshaw

Matt Fineout

David Hardie

Michael Hootman

Grzegtoz Kosmal

Naomi Langer

Mehran Mashayekh

Chris Mercier
Brent Miller

David Reddy

Marc Salette

Bruce Shepard

Rick Smith

Eva Sobesky

Derek Soltes

Todd Spiegel

Jeff Wauer

Kristin Woehl

\section{ARQUITECTO/INGENIERO EJECUTIVO IDOM}

\section{IDOM (BILBAO)}

$\begin{array}{ll}\text { César Caicoya } & \begin{array}{l}\text { Senior Architect } \\ \text { José María Asumendi } \\ \text { Puis Rodríguez } \\ \text { Jorge Garay }\end{array} \\ \text { Javier Ruiz } & \begin{array}{l}\text { Project Manager } \\ \text { Javier Mendieta }\end{array} \\ \text { Antón Amann } \\ \text { Cruz Lacoma } \\ \text { Amando Castroviejo } \\ \text { José Manuel Uribarri } \\ \text { Rogelio Díez } \\ \text { Ignacio Molinero } \\ \text { Fernando Pérez } \\ \text { Pedro Mendarozketa } \\ \text { Miguel Rodríguez } \\ \text { David Prósper } \\ \text { Javier Aróstegui } \\ \text { Víctor Zorriqueta } \\ \text { Juan José Bermejo } \\ \text { Fernando Sánchez } \\ \text { Javier Aja } \\ \text { Juan Jesús García } \\ \text { Álvaro Rey } \\ \text { Armando Bilbao } \\ \text { Gonzalo Ahumada } \\ \text { Javier Dávila } \\ \text { Imanol Múgica } \\ \text { Rafael Pérez } \\ \text { Juncal Aldamizechevarría }\end{array}$

\section{CONSULTORES EXTERNOS}

Estructura: Skidmore, Owings and Merril (Chicago).
Instalaciones: Cosentini Associates (New York).

Iluminación: LAM Partners (Boston).

Acústica: Mcway, Connant, Brook, Inc. (Los Angeles). Ernesto García Vadillo (Bilbao).

Seguridad: R. Bergámo (Italia).

Muros cortina: Peter Muller Inc. (Houston).

Sistemas constructivos: Departamento de Edificación de la Universidad de Navarra.

\section{CONTRATISTAS}

CONTRATISTAS PRINCIPALES:

DERRIBOS PETRALANDA

\section{CIMENTACIONES ABANDO}

Obras de cimentación y pilotes

U.T.E. GUGGENHEIM (FERROVIAL, URSSA, LAUKI)

Obras de Hormigón y Estructura Metálica

BALZOLA, S.A.

Obras de Cerramientos Exteriores

U.T.E. MUSEO (FERROVIAL, NORFRIO, ESTUDIOS ARRIAGA)

Obras de Cierres Interiores e Instalaciones

U.T.E. MUSEO. URBANIZACION (FERROVIAL)

Obras de Infraestructura y Acabados Exteriores

SUBCONTRATAS

\begin{tabular}{|c|c|}
\hline ABGAM & Ingeniería CATIA \\
\hline AISLAMIENTOS VASCOS & Poliuretano proyectado \\
\hline ALDAITURRIAGA & Medios auxiliares \\
\hline ARTELU & Albañilería \\
\hline AUXIMET & $\begin{array}{l}\text { Tuberías Climat. } \\
\text { Incendios/Fontanería }\end{array}$ \\
\hline BEKEA & Montajes metálicos \\
\hline BEMARSA & Aplacado de piedra \\
\hline BETTOR & Productos para hormigón \\
\hline BIKAIN & Suministro de mortero \\
\hline BOSCH TELECOM & $\begin{array}{l}\text { Seguridad/Telecomunic./nn- } \\
\text { cendios }\end{array}$ \\
\hline HORMIGONES CAVIA & Suministro de Hormigón \\
\hline CIMENTACIONES ABANDO & Cimentación \\
\hline COMPOSITES GUREA & Suelos de madera \\
\hline CONAFE & Albañilería \\
\hline CONS. BARACALDO & Ferralla \\
\hline COOPERATIVA DUMPERES & Dumper \\
\hline DILO & Montajes metálicos \\
\hline
\end{tabular}




ELDU
ELECNOR
ERKOCH
ETXEGLASS
EXCAVACIONES ARTXANDA
EXCAVACIONES JOKIN
FERRALLA BATUMAR
FOLCRA
FONTANERIA Y CALEFACCIÓN
HIPOLITO PURAS
HORMIGONES VASCOS
HORMIBAL
IMPERLEIOA
ININFOR
INSAVA
KABA
KOAL
LA BILBAINA
LA OLA
LIMPIEZAS BASAURI
LIMPIEZAS IKUSGARRI
MELCHOR LOPEZ
MYCA, S.A.L.
OLABARRIETA
PENINSULAR DE AISLAM.
PERFORACIONES Y SONDEOS
PETRALANDA
PINTURAS GALEAN
PISCITEC
POSTENSA

PROTISA

RASED

SADEKOSA

SELLADOS ALGORTA

SUESCO

TALLERES LA CASILLA

TEXSA

THYSSEN BOETTICHER

THYSSEN ROM

TOLDOS REYBLAN

U.T.E. KOOPLAD DRYWALL

ULMA

UMARAN-PERMASTEELISA

(U.T.E.)
Electricidad

Electricidad

Herrajería

Vidrios

Movimiento de tierras

Movimiento de tierras

Armaduras

Muros cortina.

Carpintería metálica

Instalaciones

Fontanería

Suministro de Hormigón

Encofrados

Impermeabilización

Topografía

Fontanería

Herrajería

Albañilería

Raseo de fachada

Carpintería de madera

Seguridad e Higiene

Seguridad e Higiene

Pintura

Escaleras metálicas

Impermeabilización

Aislamiento interior de

fibra de vidrio

Pilotaje/Cimentación

Derribos

Pintura

Estanques

Prefabricados de

Hormigón

Protección pasiva

estructura metálica

Embaldosado de

cubiertas

Impermeabilización

Sellados

Suelos de hormigón

Carpintería metálica

Impermeabilización

Ascensores

Climatización

Cierres Provisionales

Cartón yeso

Andamios

Muros Cortina, Cierre metálico, Titanio
URSSA

ICENTE ALONSO

ZAZPI

Tuberías Fontanería

Albañilería

\section{MATERIALES MÁS REPRESENTATIVOS}

Pilotes

$665 \mathrm{ud}$

Encepados

$444 \mathrm{ud}$

Hormigón

$25.760 \mathrm{~m}^{3}$

Encofrados

$58.820 \mathrm{~m}^{2}$

Armadiras

$3.256 .395 \mathrm{~kg}$

Vigas hormigón armado

$1.640 \mathrm{ml}$

Micropilotes

181 ud.

Movimiento de tierras

$85.720 \mathrm{~m}^{3}$

Placas borde ría

$1.320 \mathrm{~m}^{2}$

Estructura metálica

$4.854 .800 \mathrm{~kg}$

Protección pasiva

$68.000 \mathrm{~m}^{2}$

Rellenos

$36.490 \mathrm{~m}^{3}$

Cierre metálico y titanio

$23.530 \mathrm{~m}^{2}$

Piedra exteriores

$14.505 \mathrm{~m}^{2}$

Aislamiento exterior

$7.365 \mathrm{~m}^{2}$

Aislamiento interior

$23.280 \mathrm{~m}^{2}$

Impermeabilización con telas asfálticas

$14.950 \mathrm{~m}^{2}$

Muros cortina

$5.850 \mathrm{~m}^{2}$

Raseo exterior

$1.370 \mathrm{~m}^{2}$

Potencia calderas climat./fontanería

$3.720 \mathrm{~kW}$

Potencia eléctrica instalada

$6.500 \mathrm{kVA}$

Tuberías climat./fontanería/incendios

$37.449 \mathrm{ml}$

Cables eléctricos

$121.000 \mathrm{ml}$

Cables seguridad/telecom./incendios

$295.000 \mathrm{ml}$

Conductos climatización

$36.500 \mathrm{~m}^{2}$

$34.255 \mathrm{~m}^{2}$

$28.080 \mathrm{~m}^{2}$

$4.800 \mathrm{~m}^{2}$

$13.480 \mathrm{~m}^{2}$ 